\title{
Médiévales
}

Langues, Textes, Histoire

50 | printemps 2006

Sociétés nordiques en politique (XII ${ }^{\mathrm{e}}-\mathrm{XV}^{\mathrm{e}}$ siècles)

\section{La richesse, la sexualité et l'incitation : outils politiques des femmes dans le Nord}

Wealth, sexuality, and incitement: women's political tools in the North

Jenny Jochens

\section{(2) OpenEdition}

1 Journals

\section{Édition électronique}

URL : https://journals.openedition.org/medievales/1379

DOI : $10.4000 /$ medievales. 1379

ISSN : 1777-5892

Éditeur

Presses universitaires de Vincennes

\section{Édition imprimée}

Date de publication : 1 juin 2006

Pagination : 121-136

ISBN : 2-84292-186-0

ISSN : 0751-2708

\section{Référence électronique}

Jenny Jochens, "La richesse, la sexualité et l'incitation : outils politiques des femmes dans le Nord ", Médiévales [En ligne], 50 | printemps 2006, mis en ligne le 03 novembre 2010, consulté le 23 avril 2022 URL : http://journals.openedition.org/medievales/1379; DOI : https://doi.org/10.4000/medievales. 1379

Ce document a été généré automatiquement le 23 avril 2022.

Tous droits réservés 


\section{La richesse, la sexualité et l'incitation : outils politiques des femmes dans le Nord}

Wealth, sexuality, and incitement: women's political tools in the North

Jenny Jochens

1 Je désire traiter un sujet dont mes lecteurs pourraient dire qu'il n'existe pas : le rôle politique des femmes nordiques au Moyen Âge. Il est vrai que même si les historiens sont depuis longtemps prêts à admettre que les femmes ont bien joué des rôles dans la société comme reproductrices et productrices, c'est seulement récemment qu'on a commencé à entrevoir leur rôle politique. Mais après les travaux de Pauline Stafford, Régine le Jan, Nira Pancer et d'autres, on ne peut plus douter que les reines et les femmes de l'aristocratie ont assumé des positions importantes dans la vie politique chez les Anglo-Saxons et les Mérovingiens. En fait, Stafford a même suggéré que le $\mathrm{x}^{\mathrm{e}}$ siècle soit nommé « le siècle des reines ${ }^{1}$ ".

2 Il y a peu de femmes dans ces catégories en Europe du Nord entre le $\mathrm{x}^{\mathrm{e}}$ siècle et le XII siècle, période à laquelle je bornerai mon propos. En fait, l'Islande, d'où provient l'écrasante majorité de nos sources, n'a pas connu de reine avant 1264, date où le pays fut annexé par la Norvège. Néanmoins je crois qu'on peut bien parler d'un rôle politique des femmes mais, pour en accepter l'idée, il faut reconnaître deux conditions fondamentales, à savoir la structure de la famille germanique et le forum des affaires politiques.

3 Dans la société germanique, les familles sont organisées selon le système bilatéral où les enfants d'un couple sont considérés comme descendant également de leur père et de leur mère. Ainsi, dans certaines affaires, les filles et les femmes sont presque aussi importantes que les fils et les hommes. Il est sûr que pour avoir de l'influence, autrement dit pour faire de la politique, il faut posséder des richesses. En fait, à cause de la structure familiale, les femmes germaniques pouvaient bien être propriétaires des terres, la seule richesse qui compte dans une société agraire. 
Pour comprendre le rôle politique que les femmes ont pu exercer, il faut bien saisir le sens du mot "politique » dans les sociétés primitives et se rendre compte de l'arène où l'action se joue. Faire de la politique veut dire prendre des décisions. Un champ de bataille est sûrement une scène politique où les hommes traditionnellement prennent les décisions. Mais il $\mathrm{y}$ a des scènes plus modestes où les femmes décident seules ou en liaison avec leur mari. Du fait que les femmes possèdent des biens, la plupart des maris ne prennent pas de décisions importantes pour le ménage sans les consulter. Évidemment, il s'agit de politique sur une scène domestique, donc assez limitée, mais ce pouvoir de décision pourra s'élargir pendant le veuvage d'une femme.

\section{La richesse}

5 Nous sommes bien renseignés sur les héritages et les propriétés des femmes germaniques grâce aux lois continentales et celles des tribus nordiques². On présume que les premières furent compilées entre le $\mathrm{VI}^{\mathrm{e}}$ et le $\mathrm{VIII}^{\mathrm{e}}$ siècle tandis que les dernières sont beaucoup plus tardives. Selon ces textes, à son mariage une femme reçoit une part de son héritage parental, le plus souvent des biens mobiliers, et elle partage avec son mari les richesses acquises pendant le mariage. Néanmoins, le mari dirige tout et la femme n'obtient le contrôle de ses biens qu'au moment où elle devient veuve. À la mort de ses parents, elle reçoit des terres mais moins que ses frères. Les époux n'héritent pas l'un de l'autre mais les propriétés passeront de l'un à l'autre seulement si leurs enfants meurent sans héritiers. Ces conditions sont confirmées par les lois scandinaves mises par écrit aux XIII et $\mathrm{XIV}^{\mathrm{e}}$ siècles; comme pour les lois continentales, leurs racines se perdent dans le passé préhistorique ${ }^{3}$.

6 Heureusement, outre les lois, il existe un autre genre de sources, les inscriptions runiques, plus authentiques que les lois, compilées très tardivement, et plus fiables que les sources narratives comme les sagas. Écrites aux $\mathrm{x}^{\mathrm{e}}$ et XI $\mathrm{XI}^{\mathrm{e}}$ siècles, ces inscriptions sont en fait les seules sources vraiment authentiques de l'époque des Vikings. La plupart des pierres runiques, surtout celles de provenance suédoise, sont des commémorations des défunts érigées par les survivants. Très laconiques, elles donnent des informations fragmentées concernant les individus. S'exprimant à la troisième personne, le commanditaire s'identifie par son nom et donne aussi celui du défunt en expliquant leurs relations personnelles. Souvent, il ajoute où et comment le défunt a trouvé la mort et indique le nom du graveur. La formule est la suivante : «A érigea cette pierre après $B$, son père. $C$ cisela les runes ".

7 Les commémorations font surtout honneur aux hommes. L'écrasante majorité des commanditaires, des défunts et des graveurs sont des hommes : $93 \%$ des inscriptions honorent des hommes tandis que seulement $4 \%$ sont dédiées aux femmes et $3 \%$ célèbrent un couple marié ou d'autres groupements mixtes ${ }^{4}$. Étant donné qu'un certain nombre de pierres sont érigées pour honorer des hommes morts à l'étranger, pendant longtemps les chercheurs ont supposé que ces pierres avaient pour but de commémorer des Vikings morts pendant leurs expéditions ${ }^{5}$. Mais seulement $10 \%$ des hommes se trouvent dans cette catégorie et la conclusion s'impose que les autres ont trouvé la mort chez eux. Alors, quels sont les objectifs de ces inscriptions?

Pour répondre à cette question, il convient d'abord de se rendre compte de l'importance des liens personnels entre le commanditaire et le défunt. Dans $90 \%$ des 
inscriptions les commanditaires - hommes ou femmes - annoncent leur relation avec le défunt. Ce sont les hommes les plus proches dans la parentèle du défunt - fils, frères ou père - qui lui rendent hommage; s'il n'y en a pas, les parents plus éloignés mais toujours du sexe masculin prennent leur place - gendres, oncles, neveux. Les femmes agissaient comme commanditaires seulement en l'absence des hommes. J'accepte la thèse de Birgit Sawyer, une historienne suédoise, qui a proposé que les inscriptions soient interprétées moins comme des monuments aux morts que comme des déclarations de succession des vivants ${ }^{6}$. Autrement dit, en commémorant un mort, le commanditaire revendique publiquement les propriétés du défunt. Il est vrai que les références directes à la succession sont rares, mais les relations entre le commanditaire et le défunt sont données avec beaucoup de soin et de détails, ce qui suggère qu'il s'agit d'affaires successorales. On pourrait même se demander: quel était le but des inscriptions sinon la succession? Dans les $10 \%$ des inscriptions où les veuves commémorent leurs maris, on doit conclure qu'elles ont pu disposer de leurs richesses.

De temps en temps, la succession est mentionnée directement, surtout dans les cas de " succession aux ascendants ", situation qui arrive quand un des époux meurt après que les enfants eurent eux-mêmes déjà succombé sans héritiers; dans ce cas, l'époux survivant recueille la succession. Autrement dit, dans la succession aux ascendants, les propriétés montent à la génération précédente au lieu de descendre vers la suivante, une règle qui sûrement a favorisé plus de femmes que d'hommes. Alors, même si une femme n'hérite pas de son mari en temps normal, dans le cas précis où la femme lui survit ainsi qu'à leurs enfants sans héritiers, c'est bien ce qui se passe : les propriétés passent de la famille du défunt à la veuve.

La sphère d'influence politique d'une riche veuve pouvait s'élargir au-delà de la famille nucléaire. Les inscriptions runiques illustrent bien cette évolution. Les femmes n'ont pas utilisé leurs richesses dans le seul but de légitimer leur succession et de commémorer leur famille; peut-être encouragées par les ecclésiastiques, elles ont associé les monuments funéraires à la philanthropie en ordonnant la construction de ponts et de chaussées au bénéfice de leur communauté. En Suède, de telles constructions sont mentionnées dans une centaine d'inscriptions dont $42 \%$ étaient commanditées par des femmes ${ }^{7}$. Mais, chose significative, les femmes sont responsables de seulement $27 \%$ des inscriptions en général; cela veut dire qu'elles sont surreprésentées dans le groupe des commanditaires de ponts. Comme leurs sœurs sur le continent, les femmes nordiques ont joué ainsi un rôle culturel en promouvant la religion chrétienne; mais, tandis que dans le Sud, les femmes ont choisi de doter des églises et des monastères qui servent aussi aux donatrices elles-mêmes pendant leur veuvage et dans l'au-delà, dans le Nord, les femmes ont répondu à des besoins plus pragmatiques et séculiers - oserais-je dire politiques ? - en aidant à la construction de ponts et chaussées, utiles à l'ensemble de la société.

11 Ces buts communautaires suggèrent une société où la scène politique a déjà évolué de la famille nucléaire vers des structures plus compliquées. Il est facile de s'imaginer que de pareilles femmes - riches et de bonne famille, vivant au centre des pouvoirs émergeants - auraient pu obtenir des titres comme celui de comtesse et même de reine reflétant les positions de leur mari - le comte ou le roi - comme l'ont bien démontré pour les Mérovingiens et Carolingiens les travaux d'Emmanuelle Santinelli ${ }^{8}$.

12 La société nordique connaît peu d'exemples de femmes de ce genre. Mais cela vaut la peine de s'arrêter un moment au Danemark auprès de la reine Margareta Ingisdóttir, la 
femme du roi Nicolas (Niels) qui fut en charge du royaume pendant les trente premières années du xiie siècle (1104-1134). Fille du roi de Suède, elle fut d'abord donnée en mariage à Magnus, roi de Norvège, avec une grande dot, constituée des terres que Magnus justement avait voulu arracher de force à son père. À cette occasion elle a gagné le nom de «femme de la paix » (Friðkolla), ce qui n'a rien de surprenant. En 1103, à la mort de Magnus, elle fut mariée à Niels, recevant encore des richesses considérables. Devenue seule héritière de la Suède à la mort de son père, elle a pu créer un large réseau d'amis et d'alliés sous son contrôle personnel. Elle fut la première reine nordique du Moyen Âge à avoir son nom gravé sur les monnaies avec celui de son mari, et la première femme dont le nom fut inscrit sur une charte nordique où elle apparaissait comme témoin d'un don que son mari fit à l'église Saint-Alban à Odense ${ }^{9}$. Elle fut si importante dans les cercles royaux qu'un chroniqueur contemporain remarque :

[...] parce que le roi Niels était sans prévoyance et qu'il lui manquait la compétence nécessaire pour régner que demandent les besoins de la monarchie, l'essentiel du gouvernement pesait sur la noble reine Margareta au point que les étrangers remarquaient qu'en Danemark le gouvernement était dans les mains d'une femme ${ }^{10}$.

Ces faits et ce texte sont connus depuis longtemps mais les historiens les ont ignorés, préférant décrire le long règne du roi Niels comme une période de faiblesse pour la monarchie danoise. Ironie de l'histoire, c'est à un jeune historien suédois, Lars Hermanson, que nous devons cette nouvelle interprétation ${ }^{11}$.

\section{La sexualité}

14 Si les richesses sont importantes, pour qu'une femme puisse s'imposer, elles ne sont pas ses seuls atouts. Même une femme pauvre pouvait arriver au sommet du pouvoir pourvu qu'elle fût jeune et belle. Autrement dit, la beauté et la sexualité sont des atouts formidables dont une femme pouvait se servir à son gré. Dans les sources norroises, il y a peu d'indications que les jeunes filles aient disposé elles-mêmes de ces atouts : vivant à l'intérieur de leurs familles, protégées par leur père et leurs frères, elles avaient naturellement peu de possibilités de s'imposer ${ }^{12}$. Au contraire, c'est toujours l'homme qui prend l'initiative dans les relations entre les sexes, pour les négociations matrimoniales ou purement sexuelles. Les négociations de mariage se déroulent entièrement entre deux hommes, le marieur de la jeune fille, normalement son père, et le jeune homme qui désire l'épouser, sans qu'elle soit même consultée. Aboutissant à un contrat, ce genre d'accord est normalement paisible, mais la loi islandaise interdit et punit le rapt et autres formes de violence, en prévoyant par exemple des punitions contre les hommes qui s'introduisent travestis dans le quartier des femmes ${ }^{13}$. Pour de bonnes raisons. Suivant les sources narratives, beaucoup d'hommes manifestaient le comportement d'un Viking qui a pris l'habitude de « kidnapper les femmes belles et de bonne famille et de les garder chez lui pendant quelque temps parce que les autres hommes n'osaient pas s'y opposer ${ }^{14}$. Même si ce comportement caractérise surtout les hommes violents connus sous le terme de berserkir, souvent d'origine suédoise, beaucoup de jeunes hommes traversent une période violente avant le mariage, visitant des jeunes filles contre le gré de leur famille dans le seul but de les séduire. Fréquentes dans tous les récits, ces visites constituent un thème non négligeable de narration: elles conduisaient aux hostilités entre les deux familles, qui le plus souvent menaient à 
la mort du séducteur. "L'illicite visite d'amour» est devenue un topos dans la littérature norroise ${ }^{15}$. Le mot utilisé est le verbe fifla avec le sens d'attraper, duper ou séduire, nuances qui attribuent fermement à l'homme la responsabilité de la rencontre. $\mathrm{Au}$ commencement, la jeune fille prend parti pour sa famille, même si elle finit par accepter le séducteur. Il est clair qu'elle n'a pas d'autre choix parce que sa carrière sexuelle et reproductive est maintenant liée à lui. Bref, dans les affaires matrimoniales et sexuelles, les femmes nordiques sont plutôt passives. La seule exception concerne les femmes d'un certain âge qui tombent amoureuses d'un jeune homme, mais dans les sagas leurs tentatives de séduction ne conduisent à rien et les laissent frustrées ${ }^{16}$.

Néanmoins, la perception de la passivité sexuelle des femmes a commencé à changer avec l'introduction du christianisme. Comme ailleurs en Europe, les ecclésiastiques se sont efforcés de modifier le comportement sexuel. En Islande, les problèmes les plus graves étaient l'omniprésence du concubinage parmi les laïcs, et pour le clergé la nonobservation du célibat. L'échec de l'église sur ces deux fronts fut spectaculaire en dépit du fait qu'elle ait essayé de détourner la responsabilité des infractions de l'homme sur la femme. En ce qui concerne les laïcs, même si c'étaient les hommes qui prenaient des concubines, l'Église décida de punir les femmes plus sévèrement qu'eux. Ayant des difficultés à suivre l'obligation du célibat, les ecclésiastiques hétérosexuels ont eu bien naturellement des rêves et des fantasmes où la femme est apparue comme l'incarnation de leurs tentations. En conséquence, les prêtres ont rejeté sur les femmes la responsabilité de leurs propres faiblesses. Une histoire racontée deux fois, d'abord dans une des Sagas des familles et plus tard dans une des Sagas contemporaines, montre cette évolution. Dans une bagarre faisant suite à une "visite d'amour illicite", deux hommes perdent chacun un œil. L'évêque Ketill attaque un certain Guðmundr parce qu'il a séduit sa femme (on remarquera qu'elle était fille d'un autre évêque). Prenant au sérieux le précepte biblique « œil pour œil, dent pour dent », Guðmundr agit de même. Utilisant le mot fifldi, l'auteur de cette version rejette la responsabilité de l'affaire sur le séducteur, mais dans la version plus récente, Ketill lui-même raconte l'histoire. Même si l'affaire concerne sa propre femme, il la lui reproche en disant : « Elle ne m'était pas fidèle ${ }^{17}$ ».

16 Si les jeunes filles elles-mêmes n'ont pas reconnu le pouvoir inhérent à leur jeunesse et à leur beauté et n'ont pas su exploiter ces atouts, deux groupes d'hommes l'ont bien compris, à savoir la plupart des hommes adultes et les pères des jeunes filles. C'est sur les conditions en Islande que nous sommes le mieux renseignés. L'appétit sexuel des hommes ici est bien connu. Comme nous l'avons vu, les jeunes hommes commencent leurs exploits très tôt avec des "visites d'amour illicites ». De plus, avant son mariage, un homme prenait souvent une concubine permanente qui lui donnait des enfants. Même s'il la renvoyait avant de se marier, plus tard il prenait souvent une ou plusieurs maitresses en sus de sa femme, les maintenant toutes sous son toit. L'exemple le plus fameux concerne Jón Loptsson, le chef islandais le plus important de sa génération, qui mourut en 1197. Marié à une certaine Halldóra avec laquelle il avait deux enfants, il maintint aussi dans sa maison sa maîtresse Ragnheiðr, avec laquelle il engendra deux autres enfants, auxquels il faut ajouter trois autres issus d'autres femmes. Jón lui-même était ordonné diacre et Ragnheiðr était la sœur de Porlákr, l'évêque de Skálholt, un des deux évêchés islandais. Dorlákr essaya de réformer la vie de Jón, en ce qui concernait non seulement son mariage mais aussi ses idées sur la propriété des églises qu'il avait 
fait construire ${ }^{18}$. Le détail le plus piquant dans l'histoire est le fait que Páll, le fils de Jón et Ragnheiðr, est devenu le successeur de porlákr.

Ce comportement est aussi bien attesté dans les Sagas contemporaines dont les récits sont situés à la période chrétienne ${ }^{19}$. Curieusement, dans les Sagas des familles, qui se déroulent à l'ère païenne, les maîtresses sont rarement mentionnées. Néanmoins, on ne peut pas douter qu'elles aient été nombreuses à cette époque, même si les esclaves disparues avec le christianisme - ont aussi pu satisfaire les hommes assoiffés. Quant aux servantes et autres domestiques, elles étaient et restèrent toujours disponibles ${ }^{20}$.

Ces relations présentaient de nombreux avantages, que ce soit pour la famille d'une maitresse d'un homme important que pour la maîtresse elle-même. Au bout de quelques années, quand il s'était fatigué d'elle et après qu'elle lui eut donné quelques enfants, il était prêt à lui arranger un mariage, plus prestigieux que celui que son père aurait pu conclure pendant sa jeunesse, et il y ajoutait une dotation considérable. Dans sa nouvelle famille, la femme créait un lien important entre son mari et son ancien amant, ce qui donnait au premier des avantages politiques ${ }^{21}$. De tels bienfaits ne revenaient aux femmes qu'après s'être associées avec un homme en dehors du mariage, mais les pères étaient bien conscients des avantages qu'ils pouvaient eux-mêmes retirer de la situation, et on peut les observer exploitant le capital reproductif de leurs filles. Plus l'homme était prestigieux, plus le père était content, la prime allant naturellement aux rois nordiques. Les Sagas des rois contiennent plusieurs exemples de pères qui prenaient l'initiative de leur offrir leur fille et d'autres qui répondaient par l'affirmative quand un roi demandait de passer la nuit avec elle ${ }^{22}$.

Une fois mère, une femme non mariée avait la responsabilité de la survie de son enfant en persuadant le père de subvenir à ses besoins, non seulement dans la vie quotidienne mais aussi en lui assurant un héritage. Naturellement, l'enjeu était beaucoup plus sérieux pour les mères des garçons engendrés par des rois. En fait, partout dans le monde germanique, ces mères ont pendant plusieurs siècles joué un rôle politique considérable, essayant d'influencer la succession en faveur de leur fils. L'objectif était plus facile à atteindre pour une femme déjà installée dans l'entourage d'un chef, mais la position de maitresse était précaire et se perdait facilement. De nombreux concurrents nés de mères différentes se disputent donc la succession de leur père. Ce n'est que très tardivement que les rois du Nord ont réussi à imposer un système régulier de succession fondé sur les principes de légitimité et de primogéniture. La phase pendant laquelle plusieurs concurrents rivalisent pour régner seuls, mais où ils sont prêts à accepter, soit un gouvernement commun, soit un partage, se prolongea longtemps, notamment en Norvège. La coutume d'avoir de nombreuses partenaires sexuelles était plus commune ici qu'ailleurs et a duré plus longtemps, fortifiée par les expéditions vikings. Rarement accompagnés par femme ou amie, les hommes avaient pris l'habitude de s'associer avec des femmes autochtones, les mettant enceintes et souvent les ramenant chez eux. Vers l'an mil, quand les expéditions vikings cessèrent, ces occasions furent suspendues pour la plupart des hommes. Les rois norvégiens, au contraire, ont tardé à comprendre que leur rôle dans les mers du Nord s'était affaibli et ils continuèrent à y mener des expéditions jusqu'au milieu du XIII ${ }^{\mathrm{e}}$ siècle. Avec une femme et des maîtresses chez eux, ils établirent aussi des relations avec des femmes autochtones, dont ils eurent souvent des enfants. En conséquence, à cause du grand nombre de prétendants, pendant plus d'un siècle (1130-1240), la Norvège souffrit d'une guerre civile qui menaça l'existence même du royaume. Pas moins de quarante-six 
concurrents se présentèrent, tous prétendant à une paternité royale. Parmi eux, vingtquatre obtinrent le titre royal pour un court moment, mais seulement deux purent assurer le gouvernement dans tout le pays; douze ou peut-être treize étaient le fruit d'un mariage légitime, dont cinq obtinrent la couronne; trois revendiquaient une légitimité plus douteuse, non par leur père mais par leur mère; un des prétendants n'était pas le fils mais le frère d'un roi. Bref, un seul homme, Ingi Haraldsson, était le fils légitime d'un roi, mais il faut ajouter qu'il souffrait d'un handicap physique qui l'aurait sûrement empêché de devenir roi dans la période païenne ${ }^{23}$.

Normalement les noms des concubines royales et de leurs enfants sont connus. Si un jeune homme reste dans l'entourage du roi, ses revendications ne sont pas mises en doute. Plus difficiles sont les cas, assez nombreux, où un jeune homme arrive de l'étranger avec sa mère comme seul soutien. La mère et parfois le candidat lui-même doivent être prêts à se soumettre à l'ordalie. La mère du jeune Hákon Hákonarson, né après la mort de son père (1204), se soumit à l'épreuve, prouvant ainsi que son fils était vraiment le fils du roi Hákon avec lequel elle avait couché secrètement pendant les derniers mois de sa $v^{2}{ }^{24}$. Le cas le plus surprenant concerne le roi Sverrir, né en Norvège en 1151, apparemment le fils d'une certaine Gunnhildr et de son mari Únáss. À l'âge de cinq ans, il fut envoyé dans les îles Feroe chez le frère d'Unáss qui y était évêque. Vingt ans plus tard, lors d'un pèlerinage à Rome, Gunnhildr confessa qu'Únáss n'était pas le père de Sverrir mais que celui-ci avait été engendré par le roi Sigurðr Haraldsson, nommé Munnr (bouche). Confronté à l'affaire, le pape lui conseilla d'aller dans les îles Feroe en informer son fils. L'année suivante, Sverrir fit route vers la Norvège où il fut accepté comme roi et devint l'ancêtre d'un illustre lignage ${ }^{25}$. Les historiens ont longuement discuté de savoir si l'histoire était vraie ou si Sverrir et sa mère étaient des imposteurs (il vaut noter que Sverrir refusa de se soumettre à l'ordalie). Peu importe. Pour nous, l'histoire sert à démontrer que la revendication d'une femme fut acceptée, même si elle n'appartenait pas à l'entourage royal, et que les femmes furent capables de jouer des rôles politiques. En fait, toute la famille d'une femme associée au roi comme maîtresse ou mère jouit de son influence dans l'entourage royal ; ainsi, l'expression «le frère du roi » devint presque un titre, utilisé pour désigner un homme issu de la mère du roi mais qui n'était point de sang royal ${ }^{26}$. Ce jeu reproductif des femmes prit fin seulement avec l'acceptation vers le milieu du $\mathrm{XIII}^{\mathrm{e}}$ siècle des deux principes de monogamie et de primogéniture, réservant à une seule femme, la reine, unie au roi par un mariage monogame et fidèle, la prérogative d'engendrer son successeur.

\section{L'incitation}

21 Si une femme a ainsi utilisé ses atouts biologiques pour enfanter un fils, on peut être sûr que pour l'aider à obtenir la succession à la mort de son père, elle a dû employer des talents plus cérébraux et oratoires pour encourager l'ambition de son fils et persuader les conseillers du défunt roi de l'accepter. Bref, nous parlons maintenant de l'incitation ou du harcèlement féminins (nagging et egging, les mots équivalents en anglais, ont une connotation plus négative qu'en français parce qu'ils sont appliqués presque exclusivement aux femmes). Voici un comportement qui plonge ses racines dans le passé germanique. L'incitatrice se trouve dans le Nibelungenlied ainsi que dans la poésie des Eddas nordiques et dans la branche des sagas islandaises connue sous le titre 
de Sagas des familles, où elle est la figure féminine dominante. Provoquant les hommes mari, fils, frères, neveux et même des hommes sans aucun lien de parenté - en utilisant les mots les plus insidieux, une certaine Hetzerin - pour employer le terme allemand met en branle des événements violents pour préserver l'honneur de la famille ${ }^{27}$. Récemment, les historiens ou plutôt les historiennes ont analysé les rôles politiques des reines et des femmes de l'aristocratie dans les sociétés anglo-saxonne et mérovingienne, surtout comme donatrices et en transmettant des biens et des positions d'une génération à l'autre. Il n'est donc pas surprenant que l'on ait aussi commencé à s'intéresser à la vengeresse et à l'incitatrice. Je cite avec plaisir l'opinion de Régine Le Jan dans un livre récent: «Si l'on a souligné le rôle grandissant qu'ont pris les hommes d'Église, évêques et abbés, dans les processus de pacification, on ne s'est guère interrogé sur le rôle des femmes dans les mécanismes vindicatoires. Il y a là tout un champ de recherche qu'il faut ouvrir dans les prochaines années ${ }^{28}$. »

Les hommes ont toujours accusé les femmes de les harceler. Pierre le Chantre énumérait déjà les trois maux les plus sévères dont un homme puisse souffrir, à savoir une femme qui le harcèle, un toit qui fuit et une cheminée qui enfume ${ }^{29}$; cette liste est probablement bien antérieure au Moyen Âge. Il est vrai que de temps en temps les hommes vieux et faibles incitent d'autres hommes; on les trouve par exemple dans Beowulf et chez Saxo, mais il faut admettre que l'incitation est par excellence une activité féminine. Voilà un comportement bien intégré dans l'esprit des deux genres. Mais la figure de la Hetzerin a été renforcée pendant des siècles par des écrivains masculins: l'incitatrice est-elle un fantôme imaginaire, mais bien ancré dans la psychologie masculine, ou une réalité historique? Les sources traitant du Nord contiennent quelques allusions qui confirment l'historicité des incitatrices, non seulement dans la poésie scaldique mais aussi chez Adam de Brême et quelques autres historiographes comme Saxo Grammaticus ou encore Snorri Sturluson. Mais il faut reconnaître que la plupart des descriptions de ces femmes proviennent de sources littéraires et qu'il est difficile de les ancrer dans un contexte vraiment précis, même quand elles se trouvent dans les textes historiques tels les Sagas des rois.

Il convient de remarquer que les accusations d'incitation ne sont pas réciproques et que les hommes ne harcèlent jamais les femmes. Peut-être faudrait-il interpréter les incitations plutôt comme des cris du cœur émanant des impuissantes - les femmes adressés aux puissants - les hommes - leur demandant un partage des pouvoirs de décision en indiquant les solutions qu'elles trouvent convenables? Démontrant par leurs actions ultérieures qu'ils consentent aux demandes des femmes, par leurs mots et surtout par le langage du corps, les hommes indiquent en même temps que ces appels les ont contrariés ${ }^{30}$.

24 Le maître-créateur de l'incitatrice est Snorri Sturluson (1179-1241), mythographe, écrivain, poète, historien, homme politique, peut-être le plus célèbre Islandais de tous les temps. Il est l'auteur de l'Edda, survol de la mythologie nordique, et sûrement aussi de Heimskringla, le grand œuvre qui traite de l'histoire du monde nordique, plus spécialement de la Norvège, depuis sa création jusqu'en 1177 ; il a sans doute aussi écrit l'Egils saga, sans doute la plus célèbre des Sagas des familles, et récemment Helgi Guðmundsson a suggéré qu'il a aussi contribué à l'Orkneyinga saga ${ }^{31}$. On trouve dans ces récits un bel éventail d'incitatrices. Chose étonnante, elles sont presque sans exception placées pleinement dans la période païenne ou au moins elles ont y passé leur enfance et leur jeunesse ${ }^{32}$. Indubitablement, Snorri s'est inspiré d'une biographie d'óláfr 
Tryggvason ( $\dagger 1000)$, roi de Norvège, écrite vers 1190 par le moine Oddr Snorrason ${ }^{33}$. Dans ce livre, on trouve une esquisse assez élaborée d'une incitatrice, la fameuse Sigríðr, reine de Suède. Snorri utilise quant à lui ce nom de Sigríðr pour deux autres femmes auxquelles il attribue la responsabilité de la mort d'óláfr helgi, un autre roi norvégien, tué pendant la bataille de Stiklastaðir en 1030 alors qu'il tentait de reprendre le pouvoir. Auparavant, le roi a fait souffrir ces deux femmes, toutes deux nommées Sigríðr ; la première a perdu son mari et ses deux fils, et la seconde son fils unique sur ordre du roi. Pour se venger, les deux femmes, se servant des mots les plus insidieux et - dans le cas de la seconde Sigríðr - brandissant aussi l'épée toujours sanglante utilisée pour le meurtre de son fils, ont persuadé l'une un nouveau mari et l'autre un beau-frère de changer de camp et de se joindre aux adversaires du roi. Selon Snorri, ce sont en fait ces deux hommes qui assénèrent des coups mortels au roi pendant la bataille de 1030. Ils sont connus d'autres sources que Snorri et étaient sûrement mariés. Mais ni les noms de ces femmes ni leur rôle de harceleuses ne sont mentionnés par d'autres auteurs que Snorri. Il est possible, naturellement, qu'il ait connu des sources maintenant perdues et qu'il ait entendu des récits jamais transcrits.

Indubitablement inspiré par l'incitatrice de la poésie de l'Edda ainsi que par la figure qu'il a trouvée chez Oddr, et en même temps convaincu que beaucoup de femmes se comportent comme des incitatrices, Snorri a usé de ses dons littéraires pour créer les deux femmes nommées Sigríðr dont les figures lui ont procuré un triple avantage : tout d'abord celui de permettre d'expliquer le fait choquant qu'óláfr, le roi bien-aimé qui devint un saint immédiatement après sa mort, a été tué par les Norvégiens eux-mêmes ; ensuite celui de montrer que le revirement de l'un des deux hommes, qui a d'abord soutenu óláfr, puis s'est dressé contre lui, est dû à l'influence pernicieuse des femmes ; enfin, celui de renforcer, par l'histoire de ces deux femmes, la tension dramatique du long récit de la mort du roi.

Snorri a commencé sa grande histoire de la Norvège en écrivant la partie médiane traitant d'óláfr helgi, le saint roi. Comme je l'ai déjà indiqué, il s'est inspiré pour les deux Sigríðr de la description de la reine du même nom dans le livre d'Oddr Snorrason. Pour cette raison, il est intéressant de voir comment Snorri a traité cette reine Sigríðr, l'adversaire du roi Óláfr Tryggvason à la génération précédente. Humiliée par le refus de celui-ci de l'épouser à cause de sa religion païenne, elle jure de se venger. Mariée à Sveinn, le roi du Danemark, elle le harcèle pour l'amener à se venger d'óláfr qui avait épousé la sœur de Sveinn sans lui en demander la permission, et elle réussit à organiser contre óláfr une coalition composée de son mari et de son fils, le roi de Suède. Ne se rendant pas compte de ces intrigues, óláfr tombe dans les mains des conspirateurs à la bataille navale de Svöldr en l'an 1000, au cours d'un voyage qu'il avait entrepris dans le seul but de récupérer les propriétés considérables de sa femme byri que celle-ci avait laissées au Danemark et au Vendland. Snorri a connu ces deux femmes dans les pages d'Oddr, mais il les a considérablement modifiées. Il a amplifié le rôle incitateur de Sigríðr ; quant à byri qui, chrétienne, n'était pas une Hetzerin chez Oddr, elle l'est devenue chez Snorri. Autrement dit, Snorri a attribué à ces deux femmes la même responsabilité dans la bataille de Svöldr que celle qu'il a donnée aux deux Sigriðr dans la défaite de Stiklastaðir trente ans plus tard. Bref, à deux paires de femmes incombe la responsabilité des deux plus grands désastres qu'ait subis la Norvège au Moyen Âge, à savoir celui de Svöldr en 1000, où le roi óláfr Tryggvason disparut, et celui de Stiklastaðir en 1030, où Óláfr helgi fut tué. 
Bien d'autres incitatrices pourraient être évoquées: Gunnhildr, reine de Norvège, sur laquelle nous sommes bien renseignés et dont la longue carrière comme fille, femme et mère de rois contribue à justifier la désignation $d u \mathrm{x}^{\mathrm{e}}$ siècle comme "le siècle des reines ", sa fille Ragnhildr, femme de plusieurs chefs dans les îles Orkney, presque aussi fameuse; toutes les deux sont des personnages authentiquement historiques, connues non seulement de Snorri mais aussi d'autres auteurs. De nombreuses incitatrices sont aussi très actives dans les Sagas des familles, surtout dans la Saga de Njáll le Brûlé, la plus récente des grandes sagas classiques, qui en contient la majorité ${ }^{34}$. L'auteur de ce texte a connu les œuvres de Snorri et s'est laissé impressionner par les femmes harcelantes ; comme lui, il était convaincu que l'incitation était une activité féminine utilisable à des fins littéraires surtout quand elle se pratiquait dans le passé éloigné. Mais il faut aussi noter que dans les Sagas contemporaines, notamment la compilation connue sous le nom de Sturlunga saga, qui décrit l'histoire politique islandaise des $\mathrm{XII}^{\mathrm{e}}$ et $\mathrm{XIII}$ e siècles et fut écrite au début du XIV ${ }^{e}$ siècle, l'incitatrice est presque totalement absente. Ainsi, cette branche des sagas confirme l'impression laissée par les Sagas des rois, à savoir que la femme qui incite les hommes à la vengeance appartient bel et bien à la période païenne, mais que ce comportement est peu convenable pour une femme chrétienne. Parce qu'ils avaient peut-être conservé une mémoire plus vivace du passé païen que les auteurs continentaux, les Islandais ont pu utiliser certaines images féminines de ce passé, surtout celle de la Hetzerin, à laquelle ils ont attribué un rôle politique aux temps païens, mais on ne doit pas supposer que toutes les femmes islandaises aient joué ce rôle, et surtout pas après que la religion chrétienne eut été acceptée.

Pendant longtemps les historiens scandinaves ont maintenu que l'histoire de leur pays ne suivait pas le modèle connu ailleurs en Europe ; il y avait, au contraire, un Sonderweg scandinave, assez uniforme dans toute la région, bien que chaque pays ait pu présenter quelques variantes. C'est seulement récemment qu'ils ont découvert que, dans nombre de domaines, l'évolution en Scandinavie a suivi d'assez près l'évolution continentale. Il n'est pas surprenant que l'histoire des femmes dans le Nord se soit conformée à un nouveau modèle plus méridional. Comme nous l'avons vu, les femmes scandinaves comme leurs sœurs sur le continent - ont possédé des richesses qu'elles ont utilisées pour commémorer leurs familles et pour aider la société dans laquelle elles vivaient. Ont-elles moins bien su utiliser leur sexualité et leurs capacités reproductives à des fins personnelles et politiques ${ }^{35}$ ? Le rôle féminin le plus remarquable sur la scène politique dans le Nord serait celui tenu par la Hetzerin, mais il faut noter que cette figure se trouvait déjà chez Grégoire de Tours ${ }^{36}$. Ainsi, l'histoire des femmes aide à replacer l'histoire des sociétés nordiques dans les courants dominants de l'historiographie européenne.

\section{NOTES}

1. Voir P. STAFFORD, Queens, Concubines, and Dowagers: The King's Wife in the Early Middle Ages, Athens, Georgia, 1983; R. LE J AN, Famille et pouvoir dans le monde franc (VII $-\mathrm{X}^{e}$ siècles). Essai 
d'anthropologie sociale, Paris, 1995 ; N. PANCER, Sans peur et sans vergogne. De l'honneur et des femmes aux premiers temps mérovingiens ( $\mathrm{VI}^{e}-\mathrm{VII}^{e}$ siècles), Paris, 2001.

2. J. JOCHENS, «La femme Viking », dans Les Vikings, premiers Européens, R. BOYER éd., Paris, 2005, p. 217-32.

3. La loi islandaise appelée "Grágás » fut mise par écrit pendant l'hiver 1117-1118; les deux principaux manuscrits datent de la seconde moitié du XIII ${ }^{\mathrm{e}}$ siècle. Dans la littérature narrative, il est possible de suivre une tradition de législation royale en Norvège à partir du milieu du $\mathrm{x}^{\mathrm{e}}$ siècle, même si les lois provinciales furent mises par écrit au $\mathrm{XI}^{\mathrm{e}}$ siècle seulement. Les lois en Danemark datent de la fin de ce siècle et en Suède un siècle plus tard. Voir J. JochENS, Women in Old Norse Society, Cornell, 1995, p. 176-182.

4. B. SAWYER, Property and Inheritance in Viking Scandinavia : The Runic Evidence, Alingås, 1988.

5. L. MusSET, Introduction à la Runologie, Paris, 1965. E. MOLTKE, Runes and their origin: Denmark and elsewhere, P. Foote trad., Copenhague, 1985.

6. Voir surtout B. SAWYER, The Viking-Age Rune-Stones: Custom and Commemoration in Early Medieval Scandinavia, Oxford, 2000.

7. B. SAWYER, «Women as Bridge-Builders ; the Role of Women in Viking-Age Scandinavia ", dans People and Places in Northern Europe 500-1600, I. Wood et N. LuND éd., Woodbridge, 1991, p. 211-224.

8. Voir E. SANTINELLI, Des femmes éplorées? Les veuves dans la société aristocratique du haut Moyen Âge, Villeneuve-d'Ascq, 2003.

9. Diplomatarium Danicum, 2. 1053-1169, L. Weibull et N. SKYUM-NielSEN éd., Copenhague, 1963, p. 34. 10. S. Canutus dux, chap. 2, p. 190, dans Vitae Sanctorum Danorum, M. Cl. GERTZ éd., Copenhague, 1908-1912. Voir HERMANSON (note 11), p. 99.

11. L. HeRMANSON, Släkt, vänner och makt. En studie av elitens politiska kultur i 1100-talets Danmark, Göteborg, 2000. On rappellera que ce furent aussi deux Suédois, les frères Weibull, qui établirent la thèse qui a dominé l'historiographie danoise depuis un siècle.

12. Voir par exemple les sagas des familles. En traduction anglaise: The Complete Sagas of Icelanders, 5 vol., Reykjavík, 1997. Une sélection de cette édition se trouve dans The Sagas of Icelanders, Penguin, 2000. Sélection en français dans Sagas islandaises, R. BOYER trad., Paris, 1987.

13. Ainsi dans « Grágás ", la loi islandaise. Traduction anglaise ; voir Laws of Early Iceland : Gragas, A. DenNis, P. Foote et R. PERKINs trad., Winnipeg, 1980-2000, 2 vol.

14. L'histoire concerne un homme des Hébrides et se trouve dans Flóamanna saga, ch. 15. Une histoire similaire concernant un Islandais se trouve dans Hávarðar saga, ch. 1 ; ce texte se trouve dans Sagas islandaises, op. cit., p. 719. Voir aussi les deux frères dans Ljósvetninga saga, ch. 1.

15. J. Jochens, "The Illicit Love Visit: An Archaeology of old Norse Sexuality ", Journal of the History of Sexuality, 1, 1991, 357-392.

16. Le cas le plus frappant concerne la magicienne Katla dans Eyrbyggja saga, ch. 15-16, 20, 54 (traduction française dans Sagas islandaises, op. cit., sous le titre de « Saga de Snorri le godi »). Voir aussi J. Jochens, "Old Norse Sexuality: Men, Women, and Beasts", dans Handbook of Medieval Sexuality, V. Bullough et J. A. Brundage éd., New York, 1996, p. 369-400.

17. Les épisodes se trouvent dans Ljósvetninga saga, ch. 21 et Porgils saga ok Hafliða, ch. 28-29 dans la collection de la Sturlunga saga. Le premier se trouve en traduction anglaise dans Th. M. ANDERSSON et W. I. MilleR, Law and Literature in Medieval Iceland : « Ljósvetninga saga » and « VallaLjóts saga », Stanford, 1993, et l'autre dans La Saga des Sturlungar, R. BoYER trad., Paris, 2005 ; voir aussi J. Jochens, « The Illicit Love Visit... », loc. cit., p. 382-83.

18. L'épisode se trouve dans « Oddaverja páttr» qui fait partie d'une des versions de Porláks saga helga récemment éditée par Á. Egilsdóttir dans Biskupa sögur, II, Reykjavík, 2002 (Saga des évêques). 19. Voir par exemple le cas de Vigdís Gislsdóttir, la maîtresse de Sturla : elle fut renvoyée de la ferme de celui-ci par sa mère, avant l'arrivée de sa femme Solveig Sæmundardóttir et de sa belle- 
mère (voir La Saga des Sturlungar, op. cit., p. 282-83). Voir aussi J. JocHENS, « En Islande médiévale : à la recherche de la famille nucléaire ", Annales ESC, 40, 1980, p. 95-112.

20. Le cas le plus fameux est celui de l'esclave irlandaise Melkorka dans Laxdœela saga, ch. 12-13; pour un autre exemple, voir Grettis saga, ch. 75 (traduction française des deux exemples dans Sagas islandaises, op. cit., p. 402-406 et p. 924-926). Sur les esclaves en Scandinavie, voir R. KARRAS, Slavery and Society in Medieval Scandinavia, New Haven, 1988.

21. Voir E. EBERT, Der Konkubinat nach westnordischen Quellen, Berlin, 1993 et la recension de J. Jochens dans Alvíssmál, 3, 1994, p. 101-105.

22. Pour des exemples et leur analyse, voir J. Jochens, « The Politics of Reproduction : Medieval Norwegian Kingship », American Historical Review, 92, 1987, p. 327-349.

23. Bref aperçu en français des luttes entre prétendants au trône dans L. MUSSET, Les peuples scandinaves au Moyen Âge, Paris 1952, p. 198-209.

24. J. Jochens, Women in Old Norse Society, op. cit., p. 94-97.

25. Sverris saga etter Cod. AM 327, $4^{\circ}$, G. INDREB $\emptyset$ éd., Kristiana, 1921.

26. L'expression est konungsbróðir et qualifie un homme qui est sammœðri (" de la même mère ») d'un roi. Voir le cas de Kristrøðr, demi-frère du roi Haraldr gilli, S. STURLuson, Heimskringla, B. AĐALBJARNARSON éd., I-III, Reykjavík, 1941-1951 (Íslenzk Fornrit, 26-28), vol. 3, p. 280-281.

27. R. HelleR, Die literarische Darstellung der Frau in den Isländersagas, Halle, 1958 ; J. Jochens, Old Norse Images of Women, Philadelphia, 1996, p. 162-203.

28. R. LE JAN, Femmes, pouvoir et société dans le haut Moyen Âge, Paris, 2001, p. 20.

29. [P. Le ChANTRE], Petri Cantoris Parisiensis Verbum adbreviatum : textus conflatus, M. BoutRy éd., Turnhout, 2004, p. 764. Suivant les notes de l'éditeur, Pierre Le Mangeur, Pierre Lombard et Bernard ont également utilisé cette triade.

30. J. JocHENS, Old Norse Images of Women, op. cit., p. 189-191.

31. H. GUĐMUNDSSON, Um haf innan. Vestræenir menn og íslenzk menning á miðöldum, Reykjavík, 1997.

32. J. Jochens, "The Female Inciter in the Kings' Sagas », Arkiv för nordisk filologi, 102, 1987, p. 100-119. S. BAGGE, « Menn og kvinner i Heimskringla », Fokus på kvinner i middelalderkilder, B. J. SELLEVold, E. Mundal, G. STEINSLAND éd., Skara, 1992, p. 8-31.

33. Récemment traduite en anglais; voir The Saga of Olaf Tryggvason by Oddr Snorrason, Th. M. ANDERSSON trad., Ithaca, 2003.

34. J. JocheNs, Old Norse Images of Women, op. cit., p. 191-194 ; Saga de Njáll le Brûlé, Sagas islandaises, op. cit., p. 1203-1502.

35. Ce phénomène est mieux connu chez les rois mérovingiens. Tandis qu'au début $d u \mathrm{VI}^{\mathrm{e}}$ siècle, ces rois ont épousé des princesses de leurs voisins germaniques, dès la seconde moitié du siècle, ils commencent à prendre pour épouses des non-libres comme Austregilde, Frédegonde, Nanthilde et Bathilde, auxquelles ils donnent le statut de reine surtout si elles produisent des héritiers. Naturellement, ces femmes ont tout fait pour s'enrichir comme on le voit mieux dans le cas de Brunehilde. Voir R. LE JAN, Femmes, pouvoir et société..., op. cit., p. 15.

36. J. Jochens, Old Norse Images of Women, op. cit., p. 191-194. La magie est un autre genre où les femmes dominent ; voir EAD., « Magie et répartition entre hommes et femmes dans les mythes et la société germanico-nordiques à travers les sagas et les lois scandinaves », Cahiers de civilisation médiévale, 36, 1992, p. 375-389. 


\section{RÉSUMÉS}

Dans la société norroise, les femmes, surtout dans les classes aisées, ont pu obtenir des richesses considérables provenant de leurs parents et de leur mari grâce à un système de succession bilatéral. Même les femmes pauvres ont pu arriver au sommet du pouvoir pourvu qu'elles fussent jeunes et belles en utilisant leur sexualité et leurs capacités reproductives. Pour s'imposer, beaucoup de femmes utilisèrent aussi leurs dons de persuasion ou, comme on dit plus souvent, d'incitation. Ces trois instruments ont pu donner aux femmes du pouvoir politique dans le domaine domestique comme dans les successions royales, notamment en Norvège.

In Old Norse society women in the leading classes of society obtained considerable wealth from their native family and from husbands. Even poor women might arrive at the top of society assuming they were young and pretty by utilizing their sexual and reproductive capabilities. Most women also used their persuasive powers, their nagging and incitement, to gain their way. By using these tools women could gain considerable political power, not only on the domestic scene but also on the royal succession in Norway.

\section{INDEX}

Mots-clés : femmes norroises, inscription runiques, sagas islandaises, sexualité, succession royale

Keywords : runic inscriptions, Old Norse women, Icelandic sagas, sexuality, royal succession

\section{AUTEUR}

\section{JENNY JOCHENS}

4828 Roland Ave., Baltimore MD, 21210, USA, et 18, rue de Bièvre, F-Paris 75005 